\title{
5-Hydroxytryptamine does not reduce sympathetic nerve activity or neuroeffector function in the splanchnic circulation
}

\author{
Emma S. Darios a, Susan M. Barman a , Hakan S. Orer ${ }^{a, b, 1}$, Shaun F. Morrison c, \\ Robert P. Davis ${ }^{\mathrm{a}, 2}$, Bridget M. Seitz ${ }^{a}$, Robert Burnett ${ }^{a}$, Stephanie W. Watts ${ }^{\mathrm{a}, *}$ \\ a Department of Pharmacology and Toxicology, Michigan State University, East Lansing, Michigan USA \\ ${ }^{\mathrm{b}}$ Department of Pharmacology, Faculty of Medicine, Hacettepe University, Ankara, Turkey \\ ${ }^{c}$ Department of Neurological Surgery, Oregon Health \& Sciences University, Portland Oregon USA
}

\section{A R T I C L E I N F O}

\section{Article history:}

Received 26 November 2014

Received in revised form

9 January 2015

Accepted 17 February 2015

Available online 28 February 2015

\section{Keywords:}

5-HT

Sympathetic nerve activity

Blood pressure

\begin{abstract}
A B S T R A C T
Infusion of 5-hydroxytryptamine (5-HT) in conscious rats results in a sustained (up to 30 days) fall in blood pressure. This is accompanied by an increase in splanchnic blood flow. Because the splanchnic circulation is regulated by the sympathetic nervous system, we hypothesized that 5-HT would: 1) directly reduce sympathetic nerve activity in the splanchnic region; and/or 2) inhibit sympathetic neuroeffector function in splanchnic blood vessels. Moreover, removal of the sympathetic innervation of the splanchnic circulation (celiac ganglionectomy) would reduce 5-HT-induced hypotension. In anaesthetized Sprague-Dawley rats, mean blood pressure was reduced from $101 \pm 4$ to $63 \pm 3 \mathrm{~mm} \mathrm{Hg}$ during slow infusion of $5-\mathrm{HT}(25 \mu \mathrm{g} / \mathrm{kg} / \mathrm{min}$, i.v.). Pre- and postganglionic splanchnic sympathetic nerve activity were unaffected during 5-HT infusion. In superior mesenteric arterial rings prepared for electrical field stimulation, neither $5-\mathrm{HT}(3,10,30 \mathrm{nM})$, the $5-\mathrm{HT}_{1 \mathrm{~B}}$ receptor agonist $\mathrm{CP} 93129$ nor $5-\mathrm{HT}_{1 / 7}$ receptor agonist 5-carboxamidotryptamine inhibited neurogenic contraction compared to vehicle. 5-HT did not inhibit neurogenic contraction in superior mesenteric venous rings. Finally, celiac ganglionectomy did not modify the magnitude of fall or time course of 5-HT-induced hypotension when compared to animals receiving sham ganglionectomy. We conclude it is unlikely 5-HT interacts with the sympathetic nervous system at the level of the splanchnic preganglionic or postganglionic nerve, as well as at the neuroeffector junction, to reduce blood pressure. These important studies allow us to rule out a direct interaction of 5-HT with the splanchnic sympathetic nervous system as a cause of the 5-HT-induced fall in blood pressure.
\end{abstract}

(c) 2015 Elsevier B.V. All rights reserved.

\section{Introduction}

While 5-HT is well known as a vasoconstrictor, we found that 5-HT infused over 7-30 days in conscious rats produces a lasting reduction in mean arterial blood pressure. This was observed in multiple strains and in both male and female rats (Diaz et al., 2008; Tan et al., 2011; Davis et al., 2012, 2013). Unexpectedly, 5-HT nearly normalized arterial blood pressure in the deoxycorticosterone acetate (DOCA) salt hypertensive rat (Diaz et al., 2008). These findings

Abbreviations: 5-CT, 5-carboxamidotryptamine; 5-HT, 5-hydroxytryptamine ; 8-OH-DPAT, ( \pm )-8-hydroxy-2-dipropylaminotetralin; CP93129, 1,4-dihydro-3(1,2,3,6-tetrahydro-4-pyridinyl)-5H-pyrrol[3,2-b]pyridin-5-one; EFS, electrical field stimulation; TTX, tetrodotoxin

* Correspondence to: B445 Life Sciences Building. Department of Pharmacology and Toxicology, Michigan State University, East Lansing, MI 48824-1317. Tel.: +1517353 3724; fax: + 15173538915 .

E-mail address: wattss@msu.edu (S.W. Watts).

${ }^{1}$ Koc University School of Medicine, 34450 Istanbul, Turkey.

2 Duke University Medical Center, Durham North Carolina USA. make it important to identify the mechanism(s) by which 5-HT reduces blood pressure. 5-HT causes a robust fall in total peripheral resistance that is not caused by direct relaxation of arteries, including mesenteric arteries (Davis et al., 2012). However, microsphere studies demonstrated the ability of 5-HT to increase blood flow to the splanchnic circulation (Seitz and Watts, 2014), providing the regional focus for the current study. Since the sympathetic nervous system contributes significantly to total peripheral resistance regulation, removal of sympathetic tone is a means by which blood pressure could be reduced by $5-\mathrm{HT}$, and this is the focus of the current study. Another possibility is that 5-HT increases venous capacitance to increase blood flow, but this was not investigated presently.

There are several levels at which 5-HT could act to inhibit the function of the sympathetic nervous system. First, 5-HT can inhibit norepinephrine (NE) release at the sympathetic neuroeffector junction in blood vessels through interactions with presynaptic inhibitory 5-HT receptors (Gothert et al., 1991; Molderings et al., 1990, 2006). Second, 5-HT modifies sympathetic outflow centrally 


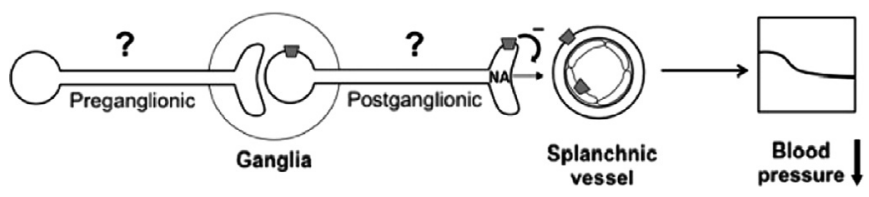

Fig. 1. Depiction of potential sites of action of 5-HT to reduce sympathetic outflow to the splanchnic bed. ?=questions examined in the present manuscript.

(Barnes and Sharp, 1999; Ramage and Villalon, 2008), reflected in changes in activity of preganglionic sympathetic nerves. Third, 5HT both decreases and increases sympathetic ganglionic activity such that postganglionic sympathetic nerve activity is influenced (Hertzler, 1961; Jones et al., 1995; Meehan and Kreulen, 1991; Pickering et al., 1994; Ramage and Villalon, 2008; Sheng-Rong et al., 1999; Watkins and Newberry, 1996).

We investigated each of these potential levels in the splanchnic circulation of the rat, depicted in Fig. 1. We hypothesized that 5-HT would: 1) reduce sympathetic nerve activity directly at either the preganglionic (potential central effect) and/or postganglionic level (ganglionic inhibition); and/or 2) directly inhibit sympathetic neuroeffector function. We used agonists of 5-HT receptors that have either been shown to acutely lower blood pressure and/or inhibit sympathetic neurotransmission. These experiments were done with an acute infusion of 5-HT. To connect these findings with the role of the sympathetic nervous system in chronic infusion of 5-HT, we examined whether removal of the primary source of sympathetic innervation to the splanchnic bed would affect the hypotension caused by 5 -HT during a one week administration.

\section{Materials and methods}

\subsection{Animal}

The Michigan State University Institutional Animal Care and Use Committee (IACUC) approved all protocols. Male Sprague Dawley rats (300-400 g, Charles River Laboratories) were used in this study.

\subsection{Methods for studies of sympathetic nerve activity}

\subsubsection{Anesthesia}

Sprague Dawley rats used for recordings of sympathetic nerve activity were anesthetized with urethane (1-1.1 g/kg i.p.) and paralyzed with gallamine triethiodide (initial dose of $20 \mathrm{mg} / \mathrm{kg}$ i.v.; supplemental doses administered as needed to maintain paralysis) following induction with isoflurane. The trachea was cannulated for maintenance of artificial respiration using positive pressure ventilation (Harvard Apparatus Inspira ASV ventilator). Rectal temperature was maintained near $37^{\circ} \mathrm{C}$ with the aid of a heat lamp.

\subsubsection{Catheterization}

Arterial and venous catheters were introduced into the femoral artery and femoral vein. The arterial catheter was connected to a pressure transducer to monitor changes in blood pressure. Venous catheter(s) were used to deliver drugs to the anesthetized rat.

\subsubsection{Nerve recording}

Potentials were recorded monophasically from the cut central ends of the preganglionic or postganglionic sympathetic splanchnic nerve placed on platinum bipolar electrodes. The capacitycoupled preamplifier bandpass was set at 1-1000 Hz. Preganglionic or postganglionic sympathetic nerve activity (SNA) was then rectified and integrated ( $1-\mathrm{V}$ reset). This signal was then quantified in 1- or 10-min data blocks at baseline and during 5-HT infusion. Hexamethonium (10 mg/kg, i.v.) was administered at the end of each experiment to verify that recordings were from preganglionic or postganglionic branches of the splanchnic nerve.

\subsection{Methods for studies involving infusion of 5-HT in conscious animals}

\subsubsection{Anesthesia}

All rats were anesthetized with isoflurane $\left(2 \%\right.$ in $\left.100 \% \mathrm{O}_{2}\right)$. Rats were treated with amoxicillin ( $150 \mathrm{mg} / \mathrm{kg} / \mathrm{i} . \mathrm{m}$.) following surgery and 3 days thereafter. All rats were treated with rimadyl $(5 \mathrm{mg} / \mathrm{kg}$, s.c. for 2 days) for general analgesia.

\subsubsection{Blood pressure probe}

Radiotelemeters (DSI PhysioTel PA series transmitter model PAC40) were implanted through subcutaneous incisions in the lower abdomen and catheters introduced into the left femoral artery. Incisions were closed with silk suture. Pressure sensing tips were advanced into the thoracic aorta. All rats were given 7 days to recover prior to any measure. Mean arterial pressure (MAP) and heart rate (HR) were recorded at 10 -min intervals (10 s recording) for the duration of the study.

\subsubsection{Alzet osmotic pump}

A small incision was made at the base of the neck. Blunt dissection was used to create a small subcutaneous pocket between the scapulae. The pump (Alzet Osmotic Pump, Model 2ML1, Duret Corporation, Cupertino, CA, $10 \mu \mathrm{l} / \mathrm{h} 7$ days) was inserted and the skin sutured closed. To each pump, a 5-HT creatinine complex [(25 $\mu \mathrm{g} / \mathrm{kg} / \mathrm{min})$ in $1 \%$ ascorbic acid as antioxidant] or vehicle (1\% ascorbic acid) was loaded. The solution was dissolved in $1 \mathrm{M} \mathrm{HCl}$, and a $\mathrm{pH}$-balance $(\sim 7)$ was achieved with $4 \mathrm{M} \mathrm{NaOH}$.

\subsection{Methods used for ganglionectomy and surgical validation}

\subsubsection{Celiac ganglionectomy}

While rats were under general anesthesia (2\% isoflurane in $100 \%$ $\mathrm{O}_{2}$ ), a ventral midline abdominal incision was performed and the small intestines were gently retracted and placed on warm saline soaked gauze. The celiac plexus located between the aorta, celiac artery and mesenteric artery was dissected free and removed (CGx). The small intestines were placed back into the abdominal cavity and lavaged with warm saline. The midline abdominal incision was sutured closed in layers. The sham group (SGx) underwent a sham operation that was performed by accessing and exposing the celiac plexus only. All rats were given an intra-muscular injection of piperacillin. Animals were used after a recovery period of five days before implantation of a 5-HT or vehicle containing pump and radiotelemeters for blood pressure measurements (described above). Rats were sacrificed by pneumothorax following deep anesthesia induced by an intraperitoneal injection of sodium pentobarbital $(60-80 \mathrm{mg} / \mathrm{kg}$, i.p.). The liver, spleen, and small intestine were dissected and stored at $-80{ }^{\circ} \mathrm{C}$ prior to isolation of amines for measurements in HPLC.

\subsubsection{High pressure liquid chromatography}

Catecholamine measures were made by homogenizing the tissue in four times their weight of $0.1 \mathrm{M}$ percholoric acid, centrifugation and taking samples through a $30 \mathrm{kDa}$ filtration tube; the filtrate was analyzed by HPLC. The HPLC system (ESA Biosciences, Chelmsford MA) consisted of a Coulochem III electrochemical detector set at $-350 \mathrm{mV}$ with separation of the analytes on an HR-80 reverse-phase column (Thermo Scientific, Waltham MA). Cat-A-Phase II (Thermo) was the mobile phase with a flow rate of $1.1 \mathrm{ml} / \mathrm{min}$ and the separation column was maintained at $35^{\circ} \mathrm{C}$. Quantification of the analytes was accomplished by performing a standard curve periodically and the limit of detection was $0.1 \mathrm{ng} / \mathrm{ml}$ for catecholamines. 
A

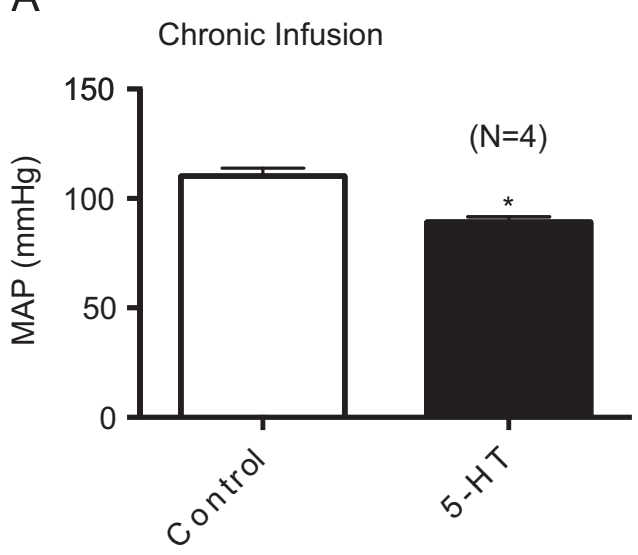

B

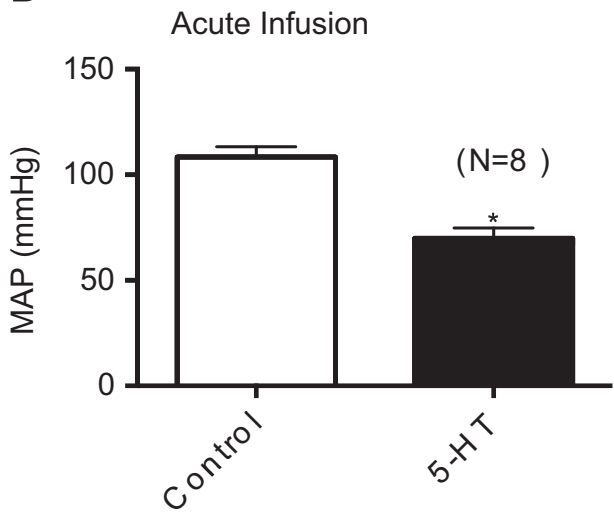

Fig. 2. 5-HT-induced fall in mean arterial blood pressure of conscious animals receiving 5-HT chronically for one week (5-HT $25 \mu \mathrm{g} / \mathrm{kg} / \mathrm{min}$; A) or anesthetized animals receiving $5-\mathrm{HT}(25 \mu \mathrm{g} / \mathrm{kg} / \mathrm{min})$ over the course of a 30-60 min infusion (B). Bars represent mean arterial pressure in normotensive Sprague Dawley rats before (control) and following 5-HT (reported at nadir of the depressor response) for the number of animals in parentheses. * statistically significant difference $(P<0.05)$ vs. appropriate control.

\subsection{Methods for electrical field stimulation}

\subsubsection{Tissue preparation}

Rats were anesthetized with pentobarbital (60 mg/kg, i.p.) and the superior mesenteric artery and vein were removed together, with in situ adipose tissue, and placed in physiological salt solution (PSS) containing (mM): $\mathrm{NaCl} 130 ; \mathrm{KCl} 4.7 ; \mathrm{KH}_{2} \mathrm{PO}_{4} 1.8 ; \mathrm{MgSO}_{4} \cdot 7 \mathrm{H}_{2} \mathrm{O}$ 1.7; $\mathrm{NaHCO}_{3}$ 14.8; dextrose 5.5; $\mathrm{CaNa}_{2}$ EDTA 0.03, $\mathrm{CaCl}_{2} 1.6$ (pH 7.2). The superior mesenteric vein was embedded in the fat around the artery. Under a microscope, the vein was carefully dissected away from the artery with fat remaining. Similarly, the artery was dissected away from the vein with fat remaining. Both vein and artery were cut into rings ( $\sim 3-5 \mathrm{~mm}$ wide) for measurement of isometric contractile force. Separate animals were used for the artery and vein experiments.

\subsubsection{Isolated tissue bath}

Two parallel stainless steel hooks were introduced into the lumen of the vessel. One segment was fixed within the warmed $\left(37{ }^{\circ} \mathrm{C}\right)$, and aerated $\left(95 \% \mathrm{O}_{2}, 5 \% \mathrm{CO}_{2}\right)$ tissue bath $(30 \mathrm{ml})$. The other was connected to a Grass isometric force transducer (FT03; Grass instruments, Quincy, MA, USA), which was connected to an ADInstruments PowerLab running Chart ${ }^{\mathbb{R}}$ version 7.0 (ADInstruments, Colorado Springs, CO.). Tissues were placed under optimal resting tension (artery: $1200 \mathrm{mg}$; vein: $400 \mathrm{mg}$ ) and allowed to equilibrate for $1 \mathrm{~h}$ before an initial challenge with a maximal concentration of phenylephrine (PE) or noradrenaline (NA; $10^{-5} \mathrm{M}$ ). After this challenge, tissues were washed until tone returned to baseline.

\subsubsection{Electrical field stimulation and isometric contraction}

Superior mesenteric artery and vein segments with surrounding fat were mounted between two platinum electrodes (positioned within the tissue bath) connected to a Grass Instruments stimulator (S88; Quincy, MA). An electrical stimulus was delivered according to the following protocol: 30 stimuli, stimulus duration $0.5 \mathrm{~ms}$, frequency $0.3-20 \mathrm{~Hz}$, voltage $14 \mathrm{~V}$. For each stimulus, isometric contractile force was measured in an isolated tissue bath as outlined above. An initial $20 \mathrm{~Hz}$ maximal stimulus was delivered to each vascular segment. To validate the neural origin of the EFS-induced contractile response, the nerve impulse propagation blocker, tetrodotoxin (TTX; $300 \mathrm{nM}$ ) was added to the tissue bath after a brief wash out period following the initial $20 \mathrm{~Hz}$ stimulus. A second $20 \mathrm{~Hz}$ stimulus was delivered to validate that contraction was abolished in the presence of TTX. In preliminary experiments, we validated that electrical field-induced contraction was sympathetically mediated as the $\alpha_{1}$ adrenergic receptor antagonist prazosin ( $50 \mathrm{nM}$ ) abolished the $20 \mathrm{~Hz}$-induced contraction. Once the neurogenic origin of contraction was established, tissues were taken through one of two protocols. First, 5-HT $\left(10^{-9}-10^{-5} \mathrm{M}\right)$ was added to the tissue baths in a cumulative fashion to construct a concentration response curve in tissues prepared for electrical field stimulation. Second, and in separate experiments, tissues were exposed to either vehicle or agonist (5-HT, 5-carboxamidotryptamine, 8-OH-DPAT, CP93129, UK14304) for 5 min prior to an electrical field stimulus. Tissues were washed three times, bringing back tension to baseline and another 5 min incubation with agonist and electric field stimulus occurred. Tissues were exposed to only one drug, building a frequency response curve in this way. Vehicle and agonist-incubated arterial segments came from the same animal.

\subsection{Materials}

Materials were purchased from US companies and solubilized in the carrier noted in parentheses. 5-carboxamidotryptamine maleate (water), 5-HT creatinine sulfate (water), ascorbate (water), gallamine, hexamethonium, ketanserin (DMSO, used dilutions in water), noradrenaline hydrochloride (water), phenylephrine hydrochloride (water) and urethane were obtained from Sigma Chemical Company (St. Louis, MO, USA). 8-OH-DPAT (water), CP93129 (water), TTX (water) and UK14304 (dimethylsulfoxide) were purchased from Tocris (R\&D systems, Minneapolis, MN, USA). Fatal Plus (Pentobarbital $390 \mathrm{mg} / \mathrm{ml}$ ) was purchased from Vortech Pharmaceutical (Dearborn MI USA). Isoflurane (IsofloTM) was purchased from Abbott (USA) and Enrofloxacin from Bayer Healthcare LLC (USA).

\subsection{Data analysis}

For sympathetic nerve activity data analysis, group differences were assessed by a two-way mixed design ANOVA and post-hoc testing at each time point was performed using Bonferroni's procedure to correct for multiple comparisons (GraphPad Prism 6). In all cases, a $P$-value of $<0.05$ was considered significant. A paired $t$-test was used to compare the level of blood pressure changes during control periods versus at the nadir during 5-HT infusion. Electrical field stimulations were expressed as a percentage of the initial $20 \mathrm{~Hz}$ maximal contraction. An unpaired students $t$-test was used to compare differences in maximal electrical field stimulated response(s) between groups. In all cases, $P<0.05$ was considered significant. All results are presented as the mean \pm S.E.M. 


\section{Results}

\subsection{The effect of 5-HT on blood pressure}

$5-\mathrm{HT}(25 \mu \mathrm{g} / \mathrm{kg} / \mathrm{min}$, Alzet pump) reduced mean arterial pressure after one week in conscious rats as measured telemetrically (Fig. 2A). Similarly, 5-HT given acutely $(25 \mu \mathrm{g} / \mathrm{kg} / \mathrm{min}$ over $30-$ $60 \mathrm{~min}$ ) caused a fall in blood pressure (Fig. 2B), supporting the idea that the mechanisms we observe in the acute situation likely applies to that of the chronic situation.

\subsection{The effect of 5-HT on sympathetic nerve activity in the splanchnic bed}

In urethane-anesthetized rats, we first investigated whether 5HT reduced sympathetic activity at the pre- and/or post-ganglionic level by recording from the splanchnic sympathetic nerve. Figs. $3 \mathrm{~A}$ and $4 \mathrm{~A}$ show representative examples of the effect of 5-HT on preganglionic and postganglionic sympathetic nerve activity in the
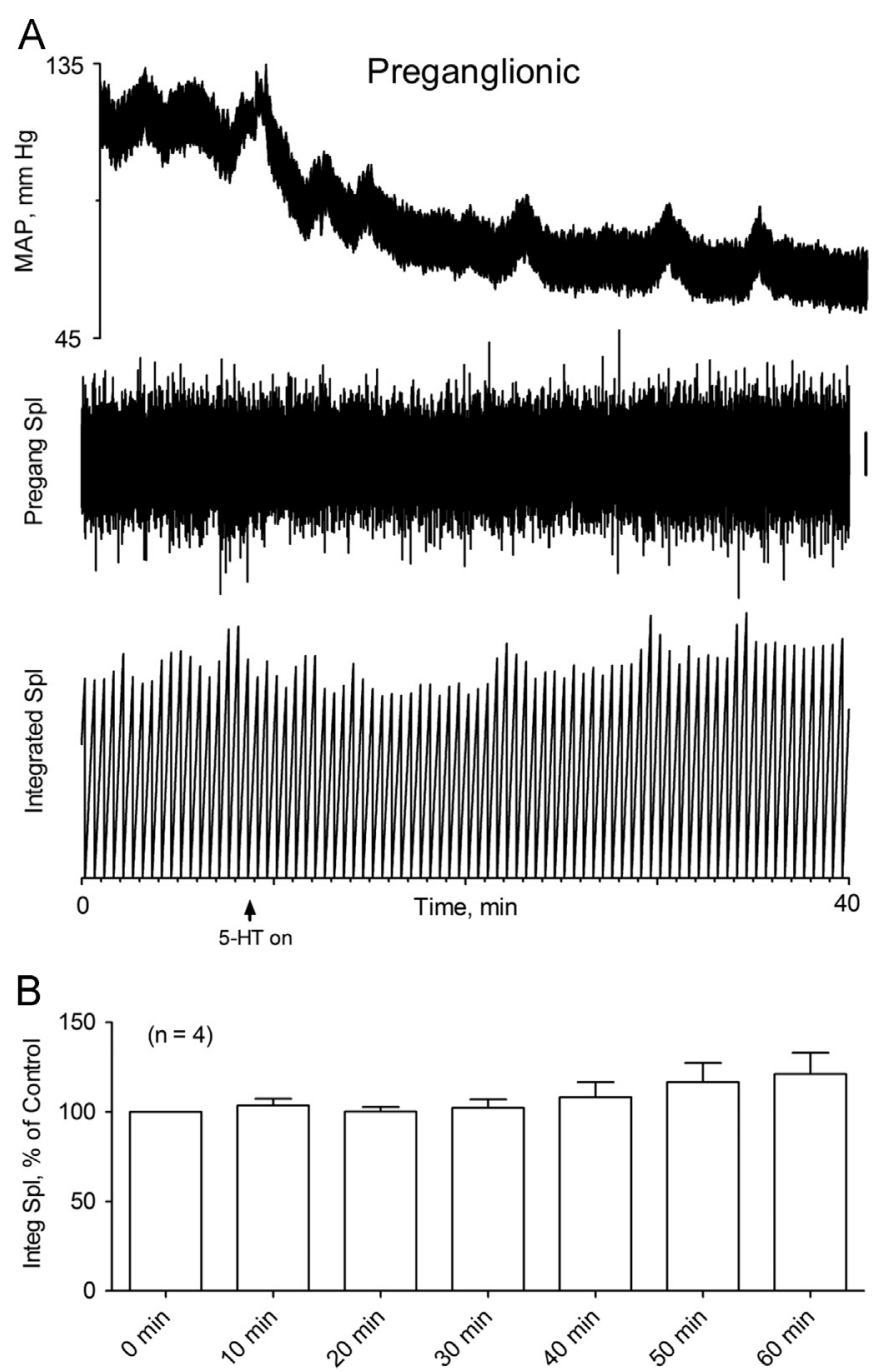

Fig. 3. Effect of acute 5-HT infusion on preganglionic sympathetic nerve activity. A: Tracing of mean arterial pressure (MAP), preganglionic sympathetic nerve activity (SNA), and integrated (Integ. Spl) preganglionic nerve activity following intravenous 5-HT infusion in the Sprague Dawley rat. Arrow marks the point at which 5-HT was delivered. B: Time course of changes in preganglionic sympathetic nerve activity (SNA) based on integrated records in the Sprague Dawley rat. Bars represent integrated preganglionic sympathetic nerve activity (mean \pm S.E.M.) at time(s) indicated following the start of intravenous 5-HT infusion for the number of animals in parentheses. splanchnic nerve, respectively. While 5-HT caused a robust fall in blood pressure in both experiments (top tracing in panel A), there was no change in integrated sympathetic nerve activity (lower tracings of panel A, integrated in panel B of each Figure). Removal of 5-HT infusion (Fig. 4A, right arrow) resulted in a rebound in blood pressure that, in some cases was accompanied by an increase in sympathetic nerve activity. In some experiments, the alpha adrenergic agonist phenylephrine (PE) was used to elicit a reflex-induced inhibition. PE robustly inhibited preganglionic activity, suggesting that we would be able to detect a 5-HTinduced inhibition of sympathetic nerve activity if it occurred.

\subsection{Effect of 5-HT on sympathetic synaptic transmission in mesenteric artery and vein}

Another potential site of action for 5 -HT is on the presynaptic terminal of the sympathetic nerve that innervates the mesenteric vasculature. Fig. 5A shares a frequency-dependent contraction in the
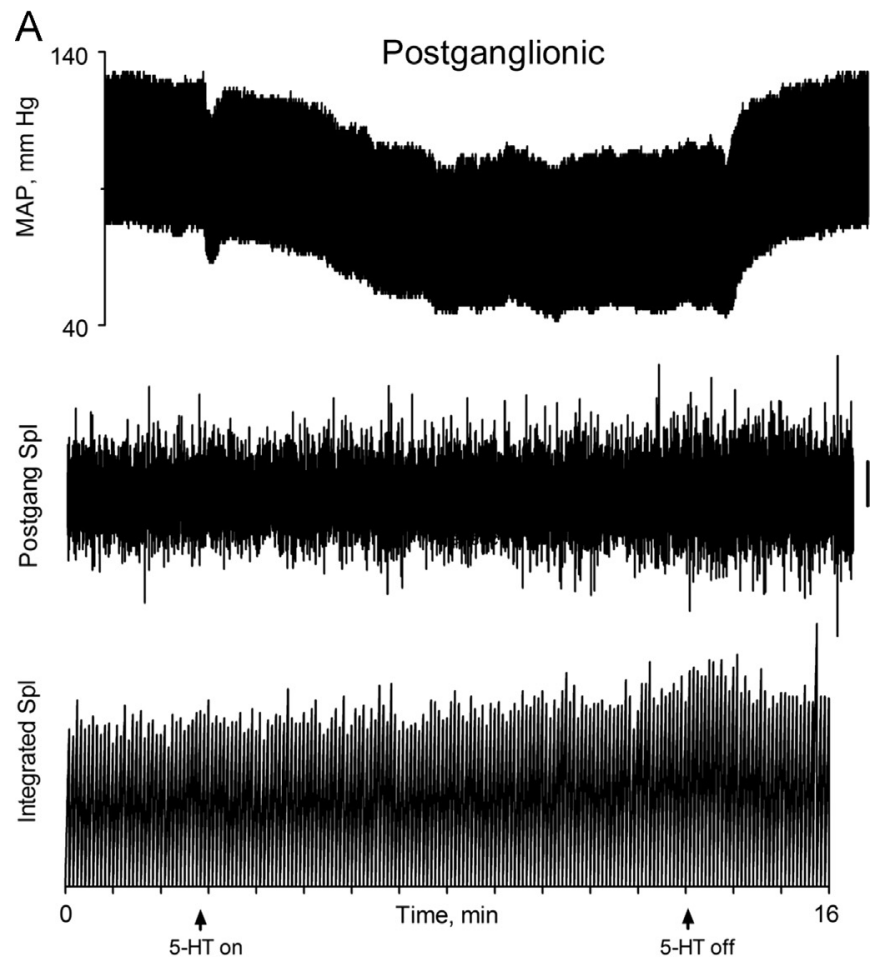

B

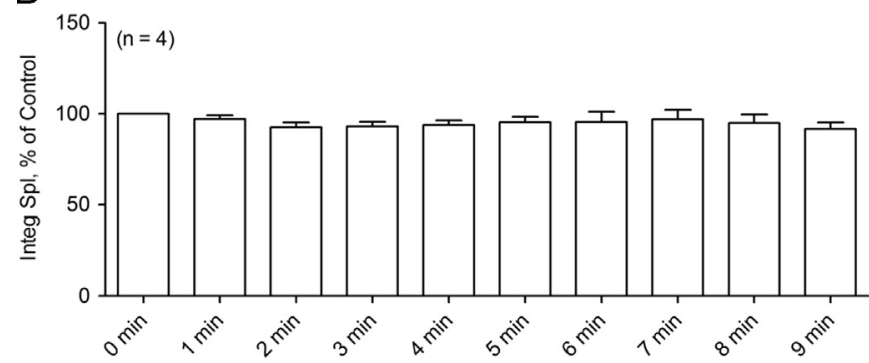

Fig. 4. Effect of acute 5-HT infusion on postganglionic sympathetic nerve activity. A. Tracing of mean arterial pressure (MAP), postganglionic splanchnic sympathetic nerve activity (SNA), and integrated (Integ. Spl) postganglionic splanchnic nerve activity following intravenous 5-HT infusion in the Sprague Dawley rat. First arrow marks the point at which 5-HT was delivered and second arrow when 5-HT infusion was stopped. B: Time course of changes in postganglionic sympathetic nerve activity (SNA) based on integrated records in the Sprague Dawley rat. Bars represent integrated postganglionic sympathetic nerve activity (mean \pm S.E.M) at time(s) indicated following the start of intravenous 5-HT infusion for number of animals indicated in parentheses. 
superior mesenteric artery (left) and baseline contraction to 5-HT (right) where the shaded concentrations that did not cause direct contraction were identified as ones to use in electric field stimulation experiments. Data in Fig. 5B supports the idea that the stimulated contraction is electrogenic as it was abolished by tetrodotoxin. Presynaptic inhibition was identifiable in our experimental protocol given the reduction of electric field stimulated contraction by the $\alpha_{2}$ adrenoceptor agonist UK14304 (Fig. 5C). Similar control experiments were done in the vein (not shown). These control experiments allowed us to proceed in testing the ability of 5-HT to inhibit electric field stimulated contraction.

At concentrations that did not directly cause contraction, 5-HT (3, 10 and $30 \mathrm{nM}$ ) did not inhibit electrical field stimulated contraction in the superior mesenteric artery when compared to vehicle (Fig. 6A). 5HT did not inhibit electrical field stimulation even with the addition of the $5-\mathrm{HT}_{2 \mathrm{~A} / 2 \mathrm{C}}$ receptor antagonist ketanserin $(50 \mathrm{nM}$ or $1 \mu \mathrm{M})$ to mask contractile receptors. Similarly, 5-HT did not modify electrical field stimulation in the presence of fluoxetine to prevent 5-HT uptake $\left(1 \mu \mathrm{M}\right.$; data not shown). A maximal concentration of the $5-\mathrm{HT}_{1 \mathrm{~B}}$ receptor agonist CP93129 was also unable to inhibit electrical field stimulated contraction (Fig. 6B); this receptor has been linked with sympathetic presynaptic inhibition. The $5-\mathrm{HT}_{1 \mathrm{~A}}$ receptor agonist $8-\mathrm{OH}-$ DPAT and 5- $\mathrm{HT}_{1 / 7}$ receptor agonist 5-carboxamidotryptamine (5-CT) were also tested as they have been linked to reduction in blood pressure. Even at a high concentration, 8-OH-DPAT did not significantly reduce stimulated contraction (Fig. 7A), nor did 8-OH-DPAT change baseline tone. 5-CT (10, $30 \mathrm{nM}$, no change in baseline contraction) did not reduce stimulated contraction (Fig. 7B). 5-HT (3-30 nM, no change in baseline contraction) also did not inhibit field-stimulated contraction in the isolated superior mesenteric vein (Fig. 7C).
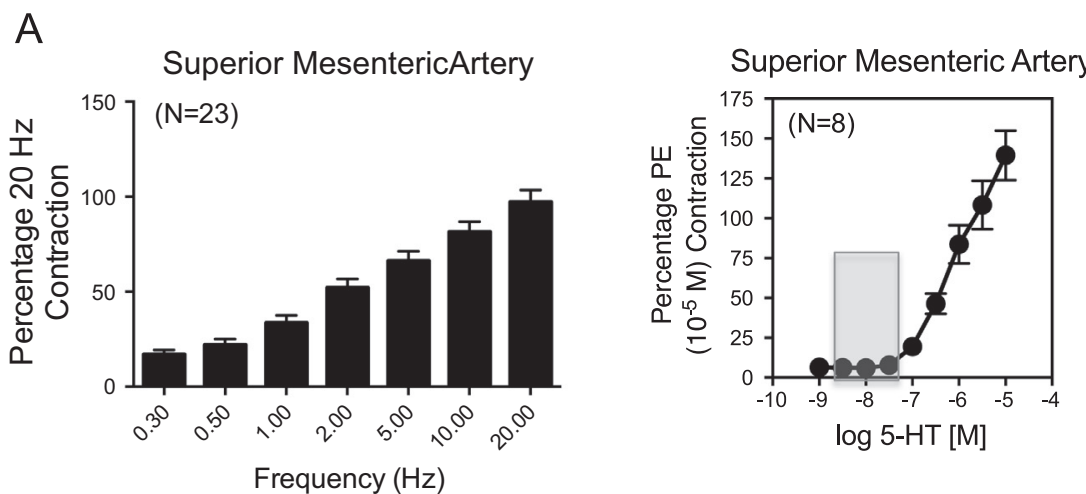

B
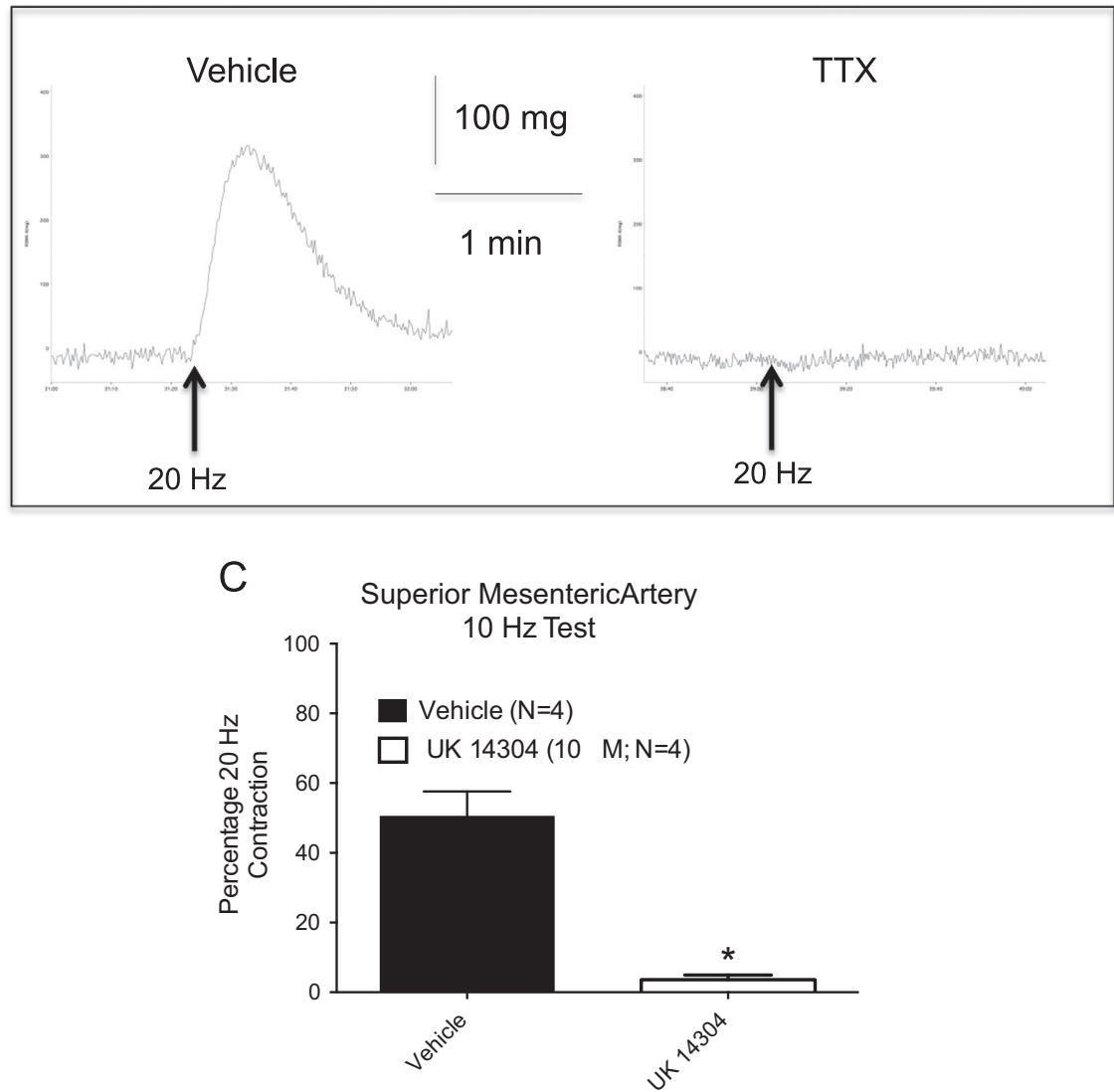

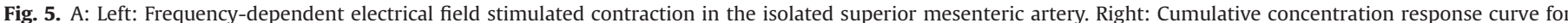

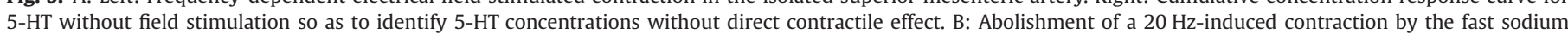

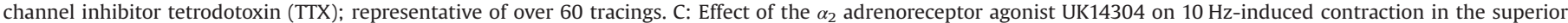
mesenteric artery. Bars/points represent mean \pm S.E.M for the number of animals in parentheses. ${ }^{*}$ signify statistically significant differences from vehicle response. 
A

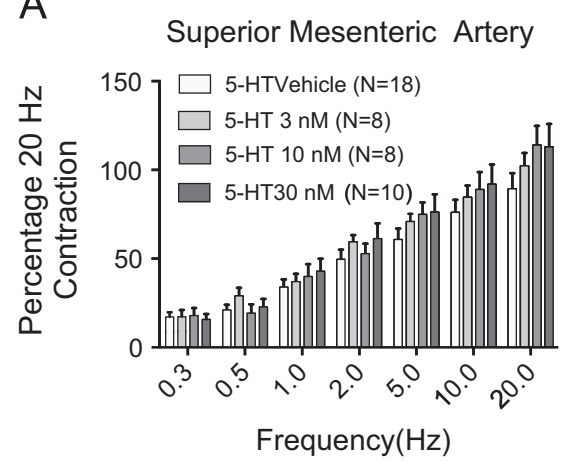

B

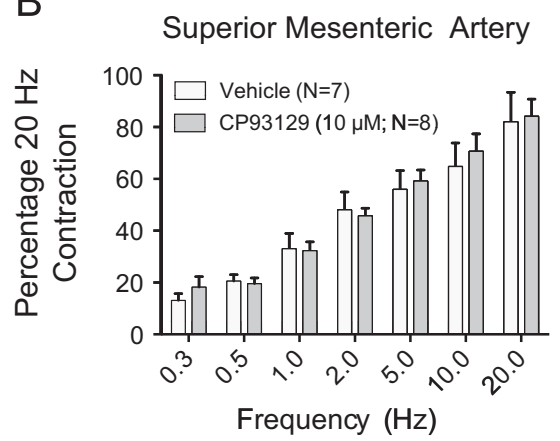

Fig. 6. Frequency-dependent $(0.3-20 \mathrm{~Hz})$ stimulated contraction in superior mesenteric arteries incubated with either vehicle $\left(\mathrm{H}_{2} \mathrm{O}\right)$ or $5-\mathrm{HT}(3,10$ and $30 \mathrm{nM}$; panel A) or the $5-\mathrm{HT}_{1 \mathrm{~B}}$ receptor agonist CP93129 $(10 \mu \mathrm{M})$ for $5 \mathrm{~min}(\mathrm{~B})$. Bars are mean \pm S.E.M for number of animals in parentheses.

\subsection{Effect of celiac ganglionectomy on 5-HT-induced hypotension}

Our last approach was to remove the primary source of sympathetic innervation of the splanchnic circulation to determine if this would prevent 5-HT from causing hypotension. The celiac ganglion provides at least part of this innervation (Czaja et al., 2002). Removal of the celiac ganglion did not change the nadir of 5-HT-induced hypotension, nor the progression of rebound in blood pressure through the week of administration when compared to sham rats (Fig. 8A). Reflexive heart rate increases are reported in Fig. 8B. Successful ganglionectomy was validated in that noradrenaline content was reduced in tissues from rats that underwent ganglionectomy compared to those with sham ganglionectomy (content $n g / g$ tissue: liver $\left[\mathrm{SGX}=49.8 \pm 14.6 ; \mathrm{CGx}=3.7 \pm 2.7^{*}\right]$, small intestine $\left[S G x=242.7 \pm 30 ; C G x=101.9 \pm 41.78^{*}\right]$, spleen $[S G x=644.8 \pm 176$; $\left.\mathrm{CGx}=1.6 \pm 0.57^{*}\right], P<0.05$ vs. SGx).

\section{Discussion}

This study took three different approaches to determine whether 5-HT caused the withdrawal of sympathetic tone from the splanchnic vasculature. Overall, our studies support the idea that a reduction in sympathetic nerve activity, at least to the splanchnic bed, does not contribute to the depressor response that accompanies an intravenous or chronic infusion of 5-HT. This valuable information helps form a direction for further studies investigating the mechanisms of 5-HT-induced hypotension.

\subsection{5-HT and inhibition of the sympathetic nervous system: direct activity measures}

Diaz et al. (2008) demonstrated that 5-HT-induced hypotension in the conscious rat was reduced by hexamethonium, a ganglionic blocker. This suggested that 5-HT may be inhibiting or causing a
A

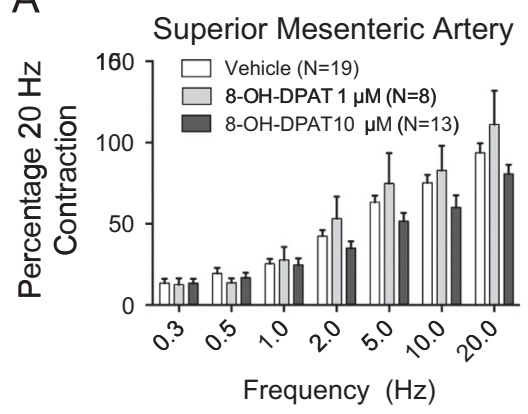

B
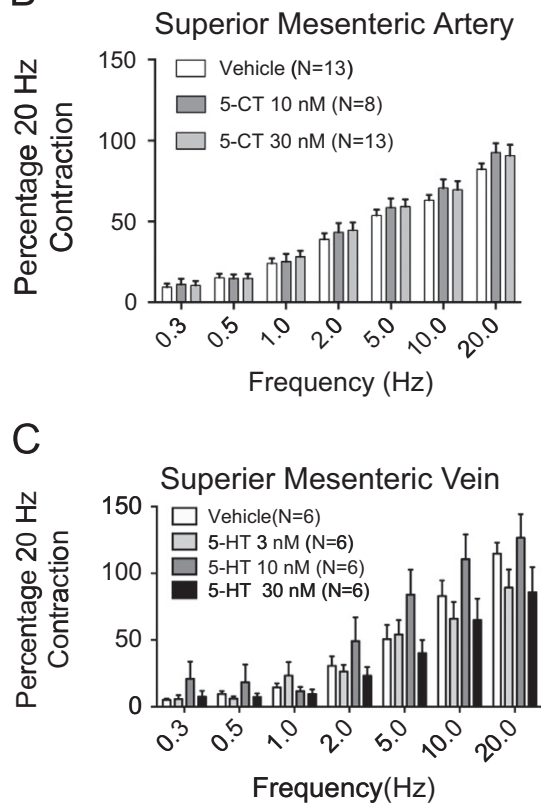

Fig. 7. Frequency-dependent $(0.3-20 \mathrm{~Hz})$ stimulated contraction in superior mesenteric arteries incubated with either vehicle $\left(\mathrm{H}_{2} \mathrm{O}\right)$ or $8-\mathrm{OH}$-DPAT $(1,10 \mu \mathrm{M}$; A) or the $5-\mathrm{HT}_{1 / 7}$ receptor agonist 5 -carboxamidotryptamine $(10,30 \mathrm{nM}$; B) for $5 \mathrm{~min}$. C. Frequency-dependent contraction in isolated mesenteric veins incubated with vehicle $\left(\mathrm{H}_{2} \mathrm{O}\right)$ or 5 - $\mathrm{HT}(3,10$ and $30 \mathrm{nM})$. Bars are mean \pm S.E.M for number of animals in parentheses.

withdrawal of sympathetic tone, such that there is less to be reduced by hexamethonium. We appreciate an alternative conclusion, namely that the effect of hexamethonium may be smaller because blood pressure has already been reduced by 5 -HT. Nonetheless, sufficient literature supports the idea that 5-HT modifies sympathetic nerve activity through ganglionic or central pathways (Hertzler, 1961; Lewis and Coote, 1990; Pickering et al., 1994). However, we did not observe a change in either pre- or post-ganglionic splanchnic sympathetic nerve activity during a slow infusion of 5-HT that was firmly associated with a fall in blood pressure. These nerves were clearly sensitive to stimuli that change blood pressure (e.g. PE) and could invoke the appropriate physiological response. This suggests that 5-HT is not acting directly at the ganglion to inhibit transmission of sympathetic outflow. These studies do not, however, permit conclusions as to whether 5-HT inhibited release of noradrenaline from sympathetic endings, and thus the next series of experiments was necessary.

\subsection{5-HT and inhibition of the sympathetic nervous system: presynaptic inhibition}

A number of conditions were employed so as to maximize the opportunity to observe interaction of 5-HT with a presynaptic receptor. While we observed robust inhibition of electrical field stimulation-induced contraction by the $\alpha_{2}$ adrenergic agonist 
A

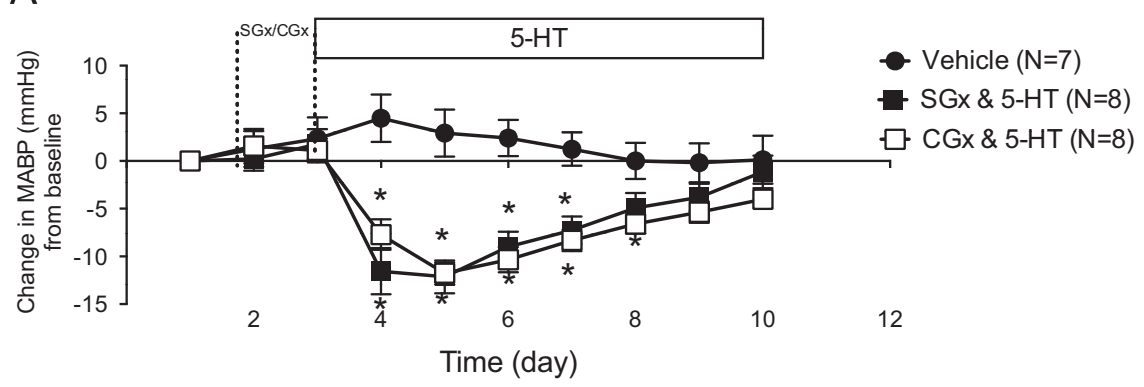

B

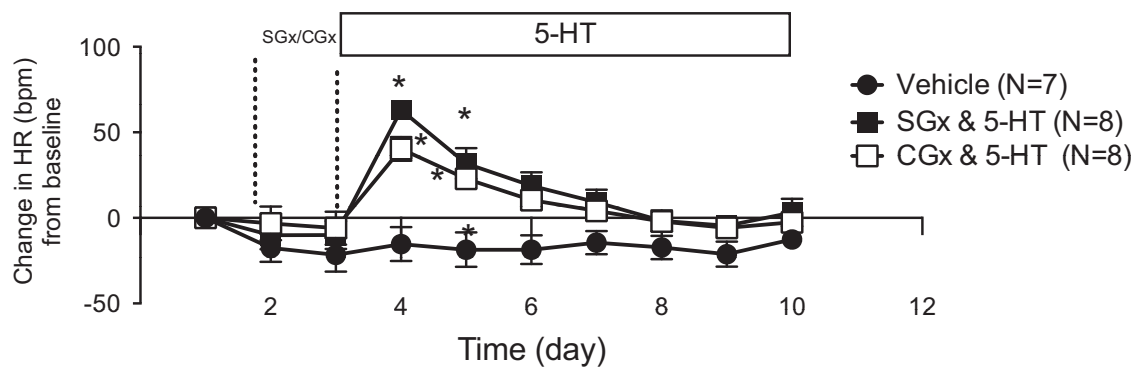

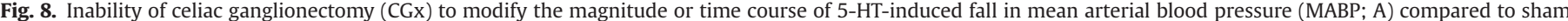

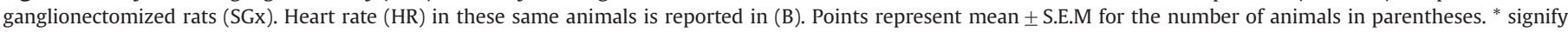
statistically significant differences from vehicle.

UK14304, inhibition was not observed, in any situation, with 5-HT in either the isolated mesenteric artery or vein. It was important to have tested 5-HT in vitro without any other interventions (e.g. antagonists, reuptake inhibitors) given that 5-HT is infused in this way into whole animals to cause a fall in blood pressure. 5-HT, through multiple 5-HT receptors, can cause presynaptic inhibition of sympathetic nerves in other blood vessels (Gothert et al., 1991; Kubo and Su, 1983; Molderings et al., 1990), and presynaptic inhibition was reviewed by Feuerstein (2008). We chose agonists of the 5-HT receptors most commonly cited for mediating presynaptic inhibition of sympathetic nerves, $5-\mathrm{HT}_{1 \mathrm{~B} / 1 \mathrm{D}}, 5-\mathrm{HT}_{1 \mathrm{~A}}$ and $5-\mathrm{HT}_{7}$ receptors, and agonists which have been associated with an acute fall in blood pressure. It should be noted that there is not a large literature relative to the effects of 5-HT on sympathetic neuroeffector junction function. The agonists used (CP93129, 8-OH-DPAT, 5-carboxamidotryptamine) were unable to inhibit electrical field stimulated contraction. 5-Carboxamidotryptamine, which has significant affinity for $5-\mathrm{HT}_{7}$ and $5-\mathrm{HT}_{1}$ receptors, was effective in inhibiting very low $(0.6 \mathrm{~Hz})$ stimulated contractions in the rabbit pulmonary artery but only above a $100 \mathrm{nM}$ concentration (Molderings et al., 2006). 5carboxamidotryptamine also caused direct contraction in the superior mesenteric artery at this concentration (not shown), so the field stimulation experiments were not performed. We did not use more selective agonists such as AS-19 to investigate the $5-\mathrm{HT}_{7}$ receptor further, given that both 8-OH-DPAT and 5-carboxoamidotryptamine were ineffective and have established affinity for the $5-\mathrm{HT}_{7}$ receptor (http://pdsp.med.unc.edu/kidb.php). In our hands, AS-19 has not proven to be efficacious in $5-\mathrm{HT}_{7}$ receptor activation, and thus we do not have confidence in its use at the present time. Our findings suggest that neither the $5-\mathrm{HT}_{1}$ nor the $5-\mathrm{HT}_{7}$ receptors are playing a role to inhibit sympathetic neuroeffector activation in the superior mesenteric artery, and the lack of efficacy of CP93129 supports this idea. We did not investigate the $5-\mathrm{HT}_{1 \mathrm{D}}$ receptor more specifically with use of an agonist such as L-694,247 because we have not been able to observe the existence of this receptor in this tissue, and published data support the $5-\mathrm{HT}_{1 \mathrm{D}}$ receptor in the rat as not being involved in sympathetic inhibition (Garcia et al., 2005; Moran et al., 2010). By contrast, a recent report by Garcia-Pedraza et al. (2013) implicates the $5-\mathrm{HT}_{1 \mathrm{D}}$ receptor in serotonergic inhibition of adrenergic neurotransmission in $5-\mathrm{HT}_{2}$ receptor blocked rats. The preponderance of evidence suggests the $5-\mathrm{HT}_{1 \mathrm{D}}$ receptor is not involved in this process.

Our work does not agree with a study by Kubo and Su (1983). They indirectly suggested that 5-HT can inhibit presynaptic receptors in the mesenteric artery because the ability of 5-HT to potentiate electrical field stimulation-induced contraction was less than that of potentiating NE-induced contraction directly. The protocols of our respective studies were too different to compare, and the interpretation of the experiments by Kubo and $\mathrm{Su}$ is arguable. Our findings suggest that 5-HT cannot inhibit presynaptic receptors in the superior mesenteric artery.

\subsection{5-HT and inhibition of the sympathetic nervous system: celiac ganglionectomy}

The last approach to testing our hypothesis was done in vivo. We removed the celiac ganglion as a source of sympathetic activity with which 5-HT might interact. Consistent with the other data in this study, removal of the celiac ganglion did not influence the progression or magnitude of 5-HT-induced hypotension. HPLC validated successful ganglionectomy by a significant reduction in the noradrenaline content of tissues known to be targets of the celiac ganglion. An alternative explanation is that a sympathetic source other than that supplied by the celiac ganglion, such as the suprarenal ganglion, contributes to sympathetic tone of the mesenteric bed. Given that removing the celiac ganglion would remove the axons from the suprarenal ganglion, it is likely that both sources were removed with the ganglionectomy performed in the present study.

\subsection{Limitations, comparisons and moving forward in understanding mechanisms of 5-HT-induced hypotension}

There are a few limitations to our study. 5-HT can exert an inhibitory influence on the sympathetic regulation of some vascular beds. 5-HT can inhibit NA release at sympathetic terminals within other blood vessels (Gothert et al., 1991; Molderings et al., 1990 2006), inhibit and facilitate transmission in autonomic ganglia (Hertzler, 1961; Jones et al., 1995; Watkins and Newberry, 1996), 
modify central sympathetic outflow (Barnes and Sharp, 1999; Ramage and Villalon, 2008) and inhibit peripheral sympathetic pressor responses in rats under a number of different conditions (Moran et al., 1994, 2010; Garcia et al., 2005; Garcia-Pedraza et al., 2013; Sanchez-Lopez et al., 2003). Our results are consistent with an inability of exogenous 5-HT to inhibit sympathetic function, at any of several levels, within the splanchnic circulation. Second, we have not taken a global measure of sympathetic nerve activity such as global noradrenaline overflow (Esler et al., 1989) or turnover. However such a measure may not be helpful if 5-HT is functioning discretely to influence a particular bed. In addition, the splanchnic bed is the only bed we studied with these multiple approaches. This choice, though, was guided by microsphere experiments that suggested that this was a site of action for 5-HT to increase flow; dilation of either venous or arterial beds could result in this observation (Seitz and Watts, 2014). However, 5-HT did not inhibit electrically-stimulated contraction in both the arterial and venous parts of splanchnic circulation. These findings guide us back to the work of De Vries et al. (1999) in considering different mechanisms by which $5-\mathrm{HT}$ elicits reduction in blood pressure. They suggest that the $5-\mathrm{HT}_{7}$ receptor mediates the 5 HT-induced decrease in arterial pressure in the vagosympathetcomized, anesthetized and ritanserin- $\left(5-\mathrm{HT}_{2}\right.$ receptor antagonist) treated rat. This particular model supports the lack of importance of the sympathetic nervous system to 5-HT-induced reduction in blood pressure but is more instrumented than our conscious model. The question becomes where the $5-\mathrm{HT}_{7}$ receptor would be located in the body to effect the hypotension. This is likely not presynaptic as the concentrations of 5-carboxytryptamine we tested have sufficient affinity for the $5-\mathrm{HT}_{7}$ receptor, and this drug was not able to inhibit electrical field stimulated contraction. Additionally, our previous work suggests it cannot be in the arterial vasculature as $5-\mathrm{HT}_{7}$ receptor agonists did not cause relaxation whether in the absence or presence of 5- $\mathrm{HT}_{2}$ receptor blockade (Davis et al., 2012). Future work will be dedicated to this conundrum.

In summary, this study suggests 5-HT is not acting to lower blood pressure in the normal rat through inhibition of noradrenaline release at the neuroeffector junction or through inhibition of pre- or postganglionic sympathetic neurons innervating the mesentery. These valuable experiments allow us to focus on other elements for mediating 5-HT-induced hypotension. The separation of 5-HT from at least splanchnic sympathetic activation, supported in this manuscript, permits focus on other mechanisms, and thus we have narrowed the possibilities by which 5-HT can act. The ultimate clinical implications of this work would be to take advantage of our mechanistic knowledge of how 5-HT reduces blood pressure and use this as a new means to reduce hypertension in the human. Blood pressure remains the primary modifiable factor for reducing the morbidity of cardiovascular disease. However, high blood pressure is underdiagnosed, normal blood pressure is achieved in only half of those with recognized hypertension, and the problem of resistant hypertension (hypertension unable to be reduced with three medications) is increasing (http://www.cdc.gov/bloodpressure/facts.htm). We are still a distance from being able to use 5-HT or precursors of 5-HT to reduce blood pressure, but a precise determination of mechanism would allow for the best design of a therapy.

\section{Funding source}

\section{NIH NHLBI HL107495.}

\section{References}

Barnes, NM, Sharp, T., 1999. A review of central 5-HT receptors and their function. Neuropharmacology 38, 1083-1152.
Czaja, K, Kraeling, R, Klimczuk, M, Franke-Radowiecka, A, Sienkiewicz, W, Lakomy, M., 2002. Distribution of ganglionic sympathetic neurons supplying the subcutaneous, perirenal and mesentery fat tissue depots in the pig. Acta Neurobiol. Exp. 62, 227-234.

Davis, RP, Pattison, J, Thompson, JM, Tiniakov, R, Scrogin, KE, Watts, SW., 2012. 5hydroxytryptamine (5-HT) reduces total peripheral resistance during chronic infusion: direct arterial mesenteric relaxation is not involved. BMC Pharmacol. 12, 4. http://dx.doi.org/10.11186/1471-2210-12-4.

Davis, RP, Szasz, T, Garver, H, Burnett, R, Tykocki, NR, Watts, SW., 2013. One-month serotonin infusion results in a prolonged fall in blood pressure in the deoxycorticosterone acetate (DOCA) salt hypertensive rat. ACS Chem. Neurosci. 4, 141-148.

De Vries, P, De Visser, PA, Heiligers, JPC, Villalón, CM, Saxena, PR., 1999. Changes in systemic and regional haemodynamics during $5-\mathrm{HT}_{7}$ receptor mediated depressor responses in rats. Naunyn-Schmiedeberg's Arch. Pharmacol. 359, 331-338.

Diaz, J, Ni, W, King, A, Fink, GD, Watts, SW., 2008. 5-hydroxytryptamine lowers blood pressure in normotensive and hypertensive rats. J. Pharmacol. Exp. Ther. 325, 1031-1038.

Esler, M, Jennings, G, Lamberg, G., 1989. Noraderenaline release and the pathophysiology of primary human hypertension. Am. J. Hypertens. 2, 140S-146S.

Feuerstein, TJ., 2008. Presynaptic receptors for dopamine, histamine and serotonin. Handb. Exp. Pharmacol. 184, 289-338.

Garcia, M, Moran, A, Calama, E, Martin, ML, Barthelmebs, M, Roman, LS., 2005. Diabetes-induced changes in 5-hydroxytryptamine inhibitory receptors involved in the pressor effect elicited by sympathetic stimulation in the pithed rat. Br. J. Pharmacol. 145, 593-601.

Garcia-Pedraza, JA, Garcia, M, Martin, ML, Gomez-Escudero, J, Rodriguez-Barbero, A, Roman, LS, Moran, A., 2013. Peripheral 5- $\mathrm{HT}_{1 \mathrm{D}}$ and 5- $\mathrm{HT}_{7}$ serotonergic receptors modulate sympathetic neurotransmission in chronic sarpogrelate treated rats. Eur. J. Pharmacol. 714, 65-73.

Gothert, M, Molderings, GJ, Fink, K, Schlicker, E., 1991. Heterogeneity of presynaptic serotonin receptors on sympathetic neurons in blood vessels. Blood Vessel. 28, 11-18.

Hertzler, EC., 1961. 5-hydroxytryptamine and transmission in sympathetic ganglia. Br. J. Pharmacol. 17, 406-413.

Jones, JFX, Martin, GR, Ramage, AG., 1995. Evidence that 5-HT 1 D receptors mediate inhibition of sympathetic ganglionic transmission in anaesthetized cats. Br. J. Pharmacol. 116, 1715-1717.

Kubo, T, Su, C., 1983. Effect of serotonin and some other neurohumoral agents on adrenergic neurotransmission in spontaneously hypertensive rat vasculature. Clin. Exp. Hypertens. A 5, 1501-1510.

Lewis, DI, Coote, JH., 1990. The influence of 5-hydroxytryptamine agonists and antagonists on identified sympathetic preganglionic neurons in the rat, in vivo, Br. J. Pharmacol. 99, 667-672.

Meehan, AG, Kreulen, DL., 1991. Electrophysiological studies on the interaction of 5hydroxytryptamine with sympathetic transmission in the guinea pig inferior mesenteric artery and ganglion. J. Pharmacol. Exp. Ther. 256, 82-87.

Molderings, GJ, Bruss, M, Gothert, M., 2006. Functional and molecular identification of 5-hydroxytryptamine receptor in rabbit pulmonary artery: involvement in complex regulation of noradrenaline release. Pharmacol. Rep. 58, 188-199.

Molderings, GJ, Werner, K, Likungu, J, Gothert, M., 1990. Inhibition of noradrenaline release from the sympathetic nerves of the human saphenous vein via presynaptic 5-HT receptors similar to the 5- $\mathrm{HT}_{1 \mathrm{D}}$ subtype. Naunyn Schmiedebergs Arch. Pharmacol. 342, 371-377.

Moran, A, Restrepo, B, de Urbina, AV, Garcia, M, Martin, ML, Roman, LS., 2010. Pharmacological profile of 5-hydroxytryptamine-induced inhibition on the pressor effect elicited by sympathetic stimulation in long term diabetic pithed rats. Eur. J. Pharmacol. 643, 70-77.

Moran, A, Velasco, C, Salvador, T, Martin, ML, San Roman, L., 1994. Inhibitory 5hydroxytryptamine receptors involved in pressor effects obtained by stimulation of sympathetic outflow from spinal cord in pithed rats. Br. J. Pharmacol. 113, 1358-1362.

Pickering, AE, Spanswick, D, Logan, SD., 1994. 5-hydroxytryptamine evokes depolarizations and membrane potential oscillations in rat sympathetic preganglionic neurons. J. Physiol. 480, 109-121.

Ramage, AG, Villalon, CM, 2008. 5-Hydroxytryptamine and cardiovascular regulation. Trends Pharmacol. Sci. 29, 472-481.

Sanchez-Lopez, A, Centurion, D, Vazquez, E, Arulmani, U, Saxena, PR, Villalon, CM., 2003. Pharmacological profile of 5-HT induced inhibition of cardioaccelerator sympathetic outflow in pithed rats. Correlation with $5-\mathrm{HT}_{1}$ and putative 5-ht5A/5B receptors. Br. J. Pharmacol. 140, 725-735.

Seitz, BM, Watts, SW., 2014. Serotonin-induced hypotension is mediated by a decrease in intestinal vascular resistance. Pharmacologica 5 (2), 50-54.

Sheng-Rong, C, Lie-Cheng, W, De-Hu, K, Zhen-Xin, H, Ru-Chun, M., 1999. Substance $\mathrm{P}$ and 5-hydroxytryptamine-mediated depolarization in sympathetic ganglion neurons. Acta Physiol. Sin. 51, 585-587.

Tan, T., Watts, S.W., Davis, R.P., 2011. Drug delivery: Enabling technology for drug discovery and development. iPRECIO micro infusion pump: programmable, refillable, and implantable. Front Pharmacol. 29 (2), 44. http://dx.doi.org/ 10.3389/fphar.2011.00044. eCollection 2011.

Watkins, CJ, Newberry, NR., 1996. Multiple 5-HT receptors in the guinea pig superior cervical ganglion. Br. J. Pharmacol. 177, 21-28. 